\title{
The QSSA in Chemical Kinetics: As Taught and as Practiced
}

\author{
Casian Pantea, Ankur Gupta, James B. Rawlings, and Gheorghe Craciun
}

\begin{abstract}
Chemical mechanisms for even simple reaction networks involve many highly reactive and short-lived species (intermediates), present in small concentrations, in addition to the main reactants and products, present in larger concentrations. The chemical mechanism also often contains many rate constants whose values are unknown a priori and must be determined from experimental measurements of the large species concentrations. A classic model reduction method known as the quasi-steady-state assumption (QSSA) is often used to eliminate the highly reactive intermediate species and remove the large rate constants that cannot be determined from concentration measurements of the reactants and products. Mathematical analysis based on the QSSA is ubiquitous in modeling enzymatic reactions. In this chapter, we focus attention on the QSSA, how it is "taught" to students of chemistry, biology, and chemical and biological engineering, and how it is "practiced" when researchers confront realistic and complex examples. We describe the main types of difficulties that appear when trying to apply the standard ideas of the QSSA, and propose a new strategy for overcoming them, based on rescaling the reactive intermediate species.

First, we prove mathematically that the program taught to beginning students for applying the 100-year-old approach of classic QSSA model reduction cannot
\end{abstract}

\footnotetext{
C. Pantea

Department of Electrical Engineering, Imperial College London, London, UK e-mail: c.pantea@imperial.ac.uk
}

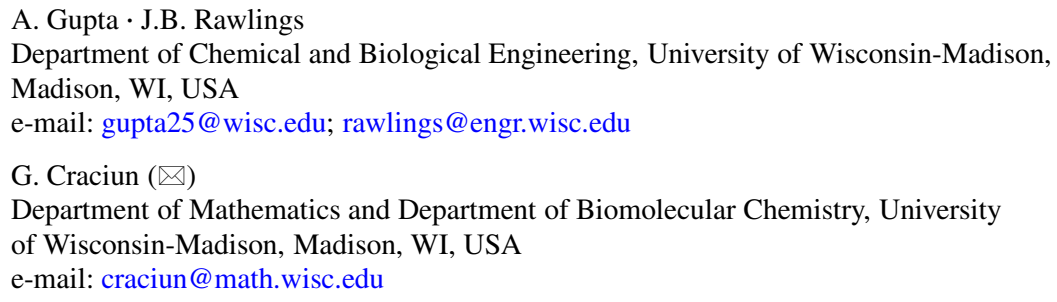


be carried out for many of the relevant kinetics problems, and perhaps even most of them. By using Galois theory, we prove that the required algebraic equations cannot be solved for as few as five bimolecular reactions between five species (with three intermediates). We expect that many practitioners have suspected this situation regarding nonsolvability to exist, but we have seen no statement or proof of this fact, especially when the kinetics are restricted to unimolecular and bimolecular reactions. We describe algorithms that can test any mechanism for solvability. We also show that an alternative to solving the QSSA equations, the Horiuti-Temkin theory, also does not work for many examples.

Of course, the reduced model (and the full model, for that matter) can be solved numerically, which is the standard approach in practice. The remaining difficulty, however, is how to obtain the values of the large kinetic parameters appearing in the model. These parameters cannot be estimated from measurements of the largeconcentration reactants and products. We show here how the concept of rescaling the reactive intermediate species allows the large kinetic parameters to be removed from the parameter estimation problem. In general, the number of parameters that can be removed from the full model is less than or equal to the number of intermediate species. The outcome is a reduced model with a set of rescaled parameters that is often identifiable from routinely available measurements. New and freely available computational software (parest_dae) for estimating the reduced model's kinetic parameters and confidence intervals is briefly described.

\section{Introduction}

The quasi-steady-state assumption (QSSA) has become a cornerstone of chemical kinetic modeling and model reduction since its introduction almost a 100 years ago $[4,5]$. Typical kinetic mechanisms describing any reasonably complex chemical system involve species that have large concentrations, namely the reactants and products, and species that have vanishingly small concentrations, usually referred to as reactive intermediates or simply intermediates. The reactive intermediates have small concentrations because their rates of formation are small compared with their rates of consumption over the range of species concentrations of interest. Models that contain highly reactive intermediates usually display a behavior with two (or more) timescales. The full model exhibits a fast timescale, during which the highly reactive intermediates change from their starting conditions (often zero) to quasi-steady values relative to the reactants and products, and a slow timescale, during which the large-concentration reactants and products evolve. The QSSA is used to remove the highly reactive, low-concentration species from the model and produce a reduced model valid on the slow timescale, which is usually the timescale of interest for analyzing measurements, identifying reduced mechanisms, estimating model parameters, and designing experiments and industrial reactors. As taught in introductory examples, the QSSA reduced model usually contains only the reactants and products. The rate expressions for the production and consumption of reactants 
and products in terms of only the reactants and products are called the reduced mechanism. Like the QSSA itself, the reduced mechanism is valid over the usual range of species concentrations exhibited by the chemical system of interest.

The quasi-steady-state assumption is widely used in modeling enzymatic reactions, where the intermediates are various enzyme-substrate complexes. The QSSA is at the core of most enzyme kinetics models, such as the Michaelis-Menten kinetics for the basic enzyme reaction $[18,20]$, the Hill kinetics for cooperative enzymatic models [11,18], models for enzyme inhibition processes [18], and the Goldbetter-Koshland function in models of futile cycles [9, 24].

The model reduction provides several advantages.

1. Model validation. The reduced mechanism allows the model developer to test the structure of the mechanism against experimental measurements. Since the reduced mechanism involves only the more easily measured high-concentration reactants and products, the experimental measurements and therefore the model validation are streamlined.

2. Parameter estimation. The full mechanism involving the reactive intermediates involves both large and small rate constants, in which the large rate constants usually correspond to the reactions that consume the intermediates. The large rate constants corresponding to intermediate consumption reactions cannot easily be identified from experimental measurements. To identify these parameters, measurements of the rapidly evolving, low-concentration intermediates are required. In the reduced model, however, these large rate constants often are removed entirely or appear as ratios to other large rate constants. This change in parametrization of the model facilitates estimating the model parameters from slow-timescale measurements of only the high-concentration reactants and products that are typically available.

3. Model solution. The evolution of species concentrations is usually described by sets of nonlinear ordinary differential equations (ODEs) that must be solved numerically. Because of the large and small rate constants, the full model's ODEs are often stiff. Even in fortuitous cases in which the large rate constants were somehow available, early ODE solvers often failed to produce accurate solutions for the stiff equations generated by the full model. ODE solvers have improved to the point that even reasonably stiff ODEs corresponding to large, complex chemical mechanisms can be solved accurately. Simplifying the mechanism and reducing the stiffness may, however, lead to a large decrease in the required computation time.

The QSSA method has a long history and a prominent place in the education of chemists, biologists, and chemical and biological engineers. Although the validity of the QSSA model as an approximation to the full mechanism has been studied extensively $[6,10,20,21]$, some fundamental questions about the method have not been addressed. In this chapter, we first explore the following unanswered question: for what class of chemical reactions can the standard procedure for applying the QSSA actually be carried out? The answer to even this basic question is surprising. 
We prove in Sect. 2 that the classic QSSA method cannot be carried out for chemical reactions as simple as those with second-order kinetics involving only three intermediates and two reactants and products. In Sect. 3, we consider two simple alternatives to the classical reduction method of the QSSA to overcome this limitation. We show that neither of these simple alternatives is sufficient for model reduction. In Sect. 4, we consider the numerical solution of the full and reduced models, and show how to rescale the species and parameters to obtain a tractable parameter estimation problem. In Sect. 6, we draw conclusions from this study and comment on future research directions.

\section{The QSSA and Its Limitations}

\subsection{The QSSA Method}

Reaction networks can involve the formation and consumption of intermediate species, which sometimes are transitory, highly reactive, and unlikely to exist outside the reaction mixture. The quasi-steady-state assumption in based on the rapid equilibration of these species. A slow-timescale model can be derived by setting the net rate of formation of these intermediates to zero. This results in a system of algebraic equations, which, if solved, provides expressions for the concentrations of the intermediates in terms of the reactant and product concentrations. Finally, this permits the construction of reaction-rate expressions for the stable reactants and stable products in terms of the reactant and product concentrations only.

We identify prospective highly reactive intermediates using characteristics such as a high rate of consumption, short lifetime, short induction time, or low concentrations. To see if a model reduction is appropriate for a given chemical mechanism, we also need to see how well the approximate solution obtained using the QSSA describes the exact solution. A detailed discussion of the selection of species in the QSSA and a list of references on this topic are given in [19].

We illustrate the QSSA approach using the following simple reaction network:

$$
\mathrm{A} \underset{k_{-1}}{\stackrel{k_{1}}{\rightleftharpoons}} 2 \mathrm{~B}, \quad \mathrm{~B} \stackrel{k_{2}}{\longrightarrow} \mathrm{C} .
$$

The corresponding system of differential equations is

$$
\begin{aligned}
& \dot{c}_{\mathrm{A}}=-k_{1} c_{\mathrm{A}}+k_{-1} c_{\mathrm{B}}^{2}, \\
& \dot{c}_{\mathrm{B}}=2 k_{1} c_{\mathrm{A}}-2 k_{-1} c_{\mathrm{B}}^{2}-k_{2} c_{\mathrm{B}}, \\
& \dot{c}_{\mathrm{C}}=k_{2} c_{\mathrm{B}} .
\end{aligned}
$$


Suppose that $k_{2} \gg k_{1}$, so that the consumption rate of $\mathrm{B}$ is large compared with its rate of production; $\mathrm{B}$ is a highly reactive intermediate and a candidate for elimination. Setting the production rate of $\mathrm{B}$ to zero gives

$$
R_{\mathrm{B}}=2 k_{1} c_{\mathrm{A}}-2 k_{-1} c_{\mathrm{B}}^{2}-k_{2} c_{\mathrm{B}}=0 .
$$

The reduced model for this example is obtained by solving this equation for $c_{\mathrm{B}}$ and substituting into the differential equations for $A$ and $C$ in (2). Here we select the single nonnegative solution for $c_{\mathrm{B}}$, but multiple nonnegative roots of QSSA algebraic systems are possible in general. In those cases, one usually selects the solution that leads to the reduced model which best fits the available data [23]. We call this procedure the standard approach to applying the QSSA. We obtain the system of differential equations

$$
\begin{aligned}
& \dot{c}_{\mathrm{A}}=-k_{1} c_{\mathrm{A}}+k_{-1}\left(\frac{-k_{2}+\sqrt{k_{2}^{2}+16 k_{1} k_{-1} c_{\mathrm{A}}}}{4 k_{-1}}\right)^{2}, \\
& \dot{c}_{\mathrm{C}}=k_{2} \cdot \frac{-k_{2}+\sqrt{k_{2}^{2}+16 k_{1} k_{-1} c_{\mathrm{A}}}}{4 k_{-1}},
\end{aligned}
$$

which corresponds to the simple reaction

$$
\mathrm{A} \stackrel{\tilde{r}}{\longrightarrow} 2 \mathrm{C}, \quad \tilde{r}=\frac{k_{2}}{2}\left(\frac{-k_{2}+\sqrt{k_{2}^{2}+16 k_{1} k_{-1} c_{\mathrm{A}}}}{4 k_{-1}}\right) .
$$

Here, the $\tilde{r}$ on top of the reaction arrow denotes a reaction rate function (and not a reaction rate constant). A comparison of the solutions to the differential equations (2) and (4) is depicted in Fig. 1. The rate constants used in the simulation were $k_{1}=k_{-1}=1, k_{2}=100$ and the initial conditions were $c_{\mathrm{A}}(0)=1, c_{\mathrm{B}}(0)=$ $c_{\mathrm{C}}(0)=0$. The two solutions are very close; the QSSA hypothesis for B is legitimate. Another lesson that is learned from model reduction by the QSSA is that complex kinetics (see the $c_{\mathrm{A}}$ dependence in the reaction rate $\tilde{r}$ ) can emerge from nothing more complicated than a few first- and second-order reactions when reactive intermediates are involved.

A key fact that allowed us to apply the QSSA was the possibility of explicitly solving equation (3) using a finite number of operations of addition, subtraction, multiplication, division, and radicals; explicit solutions of this kind are usually referred to as solutions expressible by radicals. While we are always able to solve quadratic equations explicitly, solutions expressible by radicals do not always exist for higher-degree polynomial equations. The next section reviews classical results that address this issue. 


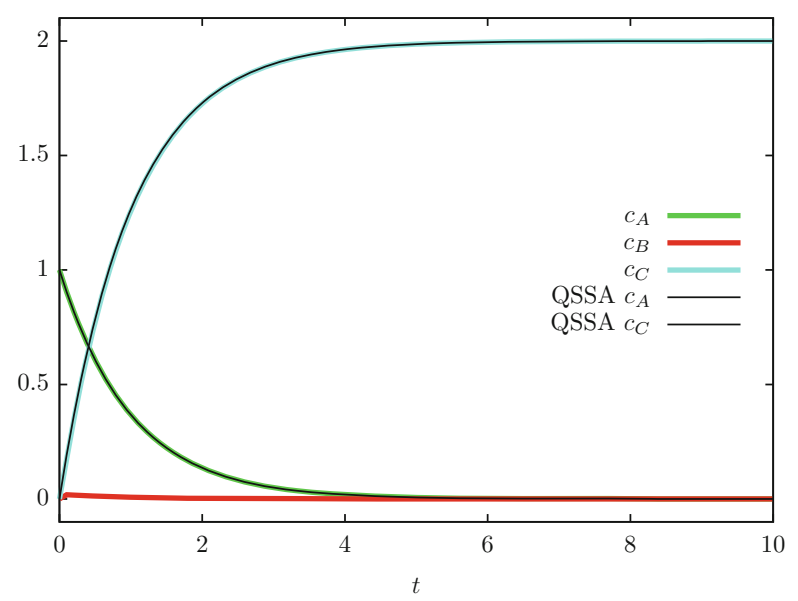

Fig. 1 The concentrations $c_{\mathrm{A}}$ and $c_{\mathrm{C}}$ with and without applying the QSSA to the intermediate B

\subsection{Solvability by Radicals}

It has been known since 1824, from the work of Niels Abel [1], that there is no formula expressible by radicals for the solution of the general fifth-degree polynomial equation. Around 1830, Évariste Galois produced his celebrated theory that gives a definitive answer to the question of solvability by radicals for any polynomial equation. In particular, this theory shows that the general polynomial equation of degree $n$ cannot be solved using radicals for any $n \geq 5$.

Here, we introduce a few notions and theorems that will allow us to use the results of Galois theory. For an excellent overview of the subject, see [15]. The notions and facts about groups and fields presented below are standard and can be found in most textbooks on abstract algebra; see, for example, [14].

Consider the general polynomial equation of degree $n$,

$$
a_{n} x^{n}+\ldots+a_{1} x+a_{0}=0,
$$

with arbitrary coefficients $a_{0}, \ldots, a_{n}$ in a field $F$. For our purposes, $F$ will be the field generated by the coefficients of a polynomial whose roots we want to find. According to the overview of the QSSA in the previous section, we need to solve for the concentrations of intermediates in terms of the concentrations of nonintermediates. Thus our coefficients are not simply elements of the field $\mathbb{Q}$ of rational numbers; they may be polynomial expressions involving variables that represent concentrations of the nonintermediate species. Consequently, our field will be the smallest field that includes all these variables. This field is usually denoted by $\mathbb{Q}\left(c_{1}, \ldots, c_{l}\right)$, the set of quotients of real polynomials in the variables $c_{1}, \ldots, c_{l}$, representing the concentrations of the nonintermediate species. For instance, in the 
example represented by Eq. (2), to find the concentration $c_{\mathrm{B}}$ we solved $2 k_{1} c_{\mathrm{A}}-$ $2 k_{-1} c_{\mathrm{B}}^{2}-k_{2} c_{\mathrm{B}}=0$, viewed as an equation in $c_{\mathrm{B}}$ with coefficients in $\mathbb{Q}\left(c_{\mathrm{A}}\right)$.

If the roots of Eq. (5) are not in $F$, there always exists a larger field that contains all these roots; let us denote these roots by $x_{01}, \ldots, x_{0 n}$. The set of polynomials with variables $x_{1}, \ldots x_{n}$ and coefficients in the field $F$ is denoted by $F\left[x_{1}, \ldots, x_{n}\right]$. A polynomial $Q$ in $F\left[x_{1}, \ldots, x_{n}\right]$ that vanishes at the point $\left(x_{01}, \ldots, x_{0 n}\right)$ is called a relation among the roots of (5). Recall that the symmetric group $S_{n}$ is the group of all permutations of $n$ distinct elements.

Definition 1. The Galois group of the algebraic equation (5) over the field $F$ is the subgroup of the symmetric group $S_{n}$ consisting of the permutations of the roots $\left(x_{01}, \ldots, x_{0 n}\right)$ that preserve all the relations among these roots.

Galois theory introduces the key notion of a solvable group; for a detailed description of this notion, see [14]. The following theorem is the main result regarding solvability of algebraic equations by radicals.

Theorem 1. An algebraic equation of the form (5) is solvable by radicals if and only if its Galois group is solvable.

Therefore, the question of whether or not the solution of an algebraic equation can be made explicit using radicals is equivalent to checking a certain property of a group involving the coefficients of the equation. Most modern computer algebra software can handle the latter problem; we find the software package Maple [17] particularly suitable for this task.

It can be shown that the Galois group of the general polynomial equation of degree $n$ with arbitrary coefficients is $S_{n}$. In view of Theorem 1 , the nonsolvability by radicals of the general polynomial equation of degree $\geq 5$ is then explained by the following result.

Theorem 2. The symmetric group $S_{n}$ is solvable if and only if $n<5$.

Before concluding this section, we shall mention another algebraic notion that we will use. Roughly speaking, a Groebner basis replaces a system of polynomial equations for $n$ variables by a "nicer" one which has the same set of solutions as the first. For example, if the original system has a finite number of solutions (which will usually be the case for systems arising from chemical kinetics), we can choose a Groebner basis where one of the equations is a univariate polynomial in one of the variables. This way, we are able to address the problem of the explicit solvability of the initial multivariate polynomial system by using Galois theory on the univariate polynomial in the Groebner basis. A standard reference on Groebner bases is [7].

\subsection{Nonsolvable Examples}

As we saw above, a key step in the standard QSSA approach is solving a certain system of polynomial equations. But this is not always possible, and thus we are 
confronted with an important limitation of this approach to applying the QSSA. In what follows, we present two chemical reaction networks where the standard QSSA approach cannot be used because of the nonsolvability by radicals of the resulting systems of algebraic equations.

The first example consists of the following mechanism:

$$
\begin{aligned}
& 2 \mathrm{Y} \underset{k_{-1}}{\stackrel{k_{1}}{\rightleftharpoons}} 2 \mathrm{~B}, \\
& \mathrm{Y}+\mathrm{B} \stackrel{k_{2}}{\longrightarrow} \mathrm{Z}+\mathrm{A}, \\
& \mathrm{Z}+\mathrm{B} \underset{k_{-3}}{\stackrel{k_{3}}{\rightleftharpoons}} 2 \mathrm{X}, \\
& \mathrm{A}+\mathrm{X} \stackrel{k_{4}}{\longrightarrow} \mathrm{Y}+\mathrm{B}, \\
& 2 \mathrm{Z} \stackrel{k_{5}}{\longrightarrow} 2 \mathrm{~A} \text {, }
\end{aligned}
$$

whose dynamics is given by the equations

$$
\begin{aligned}
& \dot{c}_{\mathrm{A}}=k_{2} c_{\mathrm{B}} c_{\mathrm{Y}}-k_{4} c_{\mathrm{A}} c_{\mathrm{X}}+2 k_{5} c_{\mathrm{Z}}^{2}, \\
& \dot{c}_{\mathrm{B}}=2 k_{1} c_{\mathrm{Y}}^{2}-2 k_{-1} c_{\mathrm{B}}^{2}-k_{2} c_{\mathrm{B}} c_{\mathrm{Y}}-k_{3} c_{\mathrm{B}} c_{\mathrm{Z}}+k_{-3} c_{\mathrm{X}}^{2}+k_{4} c_{\mathrm{A}} c_{\mathrm{X}}, \\
& \dot{c}_{\mathrm{X}}=2 k_{3} c_{\mathrm{B}} c_{\mathrm{Z}}-2 k_{-3} c_{\mathrm{X}}^{2}-k_{4} c_{\mathrm{A}} c_{\mathrm{X}}, \\
& \dot{c}_{\mathrm{Y}}=-2 k_{1} c_{\mathrm{Y}}^{2}+2 k_{-1} c_{\mathrm{B}}^{2}-k_{2} c_{\mathrm{B}} c_{\mathrm{Y}}+k_{4} c_{\mathrm{A}} c_{\mathrm{X}}, \\
& \dot{c}_{\mathrm{Z}}=k_{2} c_{\mathrm{B}} c_{\mathrm{Y}}-k_{3} c_{\mathrm{B}} c_{\mathrm{Z}}+k_{-3} c_{\mathrm{X}}^{2}-2 k_{5} c_{\mathrm{Z}}^{2} .
\end{aligned}
$$

To obtain fast equilibration of $\mathrm{X}, \mathrm{Y}$, and $\mathrm{Z}$, we choose $k_{1}, k_{-3}, k_{5}$ to be "large", $k_{-1}, k_{4}$ to be "small", and $k_{2}, k_{3}$ to be of order $O(1)$. Then the species X, Y, and $\mathrm{Z}$ are fast intermediates, and are candidates for the QSSA approach. Therefore, we need to solve the following system of algebraic equations for $c_{\mathrm{X}}, c_{\mathrm{Y}}$, and $c_{\mathrm{Z}}$ :

$$
\begin{aligned}
2 k_{3} c_{\mathrm{B}} c_{\mathrm{Z}}-2 k_{-3} c_{\mathrm{X}}^{2}-k_{4} c_{\mathrm{A}} c_{\mathrm{X}} & =0, \\
-2 k_{1} c_{\mathrm{Y}}^{2}+2 k_{-1} c_{\mathrm{B}}^{2}-k_{2} c_{\mathrm{B}} c_{\mathrm{Y}}+k_{4} c_{\mathrm{A}} c_{\mathrm{X}} & =0, \\
k_{2} c_{\mathrm{B}} c_{\mathrm{Y}}-k_{3} c_{\mathrm{B}} c_{\mathrm{Z}}+k_{-3} c_{\mathrm{X}}^{2}-2 k_{5} c_{\mathrm{Z}}^{2} & =0 .
\end{aligned}
$$

We have used the software package Maple to generate a Groebner basis of this algebraic system. To facilitate the computation, we chose all the "large" reaction constants to be equal to $K$, the "small" ones equal to $k$, and all other reaction constants to be 1 . An element of this basis was then found to be the univariate polynomial

$$
\begin{aligned}
& P\left(c_{\mathrm{Z}}\right)=32 k^{2} c_{\mathrm{B}}^{8} K-4 k^{3} c_{\mathrm{B}}^{6} c_{\mathrm{A}}^{2}-2 k^{5} c_{\mathrm{B}}^{4} c_{\mathrm{A}}^{4}+ \\
&\left(-k^{4} c_{\mathrm{A}}^{4} c_{\mathrm{B}}^{3}-2 k^{2} c_{\mathrm{A}}^{2} c_{\mathrm{B}}^{5}-16 k^{3} K c_{\mathrm{B}}^{5} c_{\mathrm{A}}^{2}\right) c_{\mathrm{Z}}+
\end{aligned}
$$




$$
\begin{aligned}
& \left(32 k^{3} K^{2} c_{\mathrm{B}}^{4} c_{\mathrm{A}}^{2}+4 k^{4} c_{\mathrm{A}}^{4} c_{\mathrm{B}}^{2}-64 k K^{2} c_{\mathrm{B}}^{6}+4 k^{2} K c_{\mathrm{A}}^{2} c_{\mathrm{B}}^{4}\right) c_{\mathrm{Z}}^{2}+ \\
& \left(8 K^{2} k^{4} c_{\mathrm{A}}^{4} c_{\mathrm{B}}+48 k^{2} K^{2} c_{\mathrm{B}}^{3} c_{\mathrm{A}}^{2}\right) c_{\mathrm{Z}}^{3}+ \\
& \left(32 K^{3} c_{\mathrm{B}}^{4}-16 K^{3} k^{2} c_{\mathrm{A}}^{2} c_{\mathrm{B}}^{2}+8 K^{3} k^{4} c_{\mathrm{A}}^{4}-256 k K^{4} c_{\mathrm{B}}^{4}\right) c_{\mathrm{Z}}^{4}- \\
& 64 K^{4} k^{2} c_{\mathrm{A}}^{2} c_{\mathrm{B}} c_{\mathrm{Z}}^{5}+ \\
& \left(-128 K^{5} k^{2} c_{\mathrm{A}}^{2}+256 K^{5} c_{\mathrm{B}}^{2}\right) c_{\mathrm{Z}}^{6}+ \\
& 512 K^{7} c_{\mathrm{Z}}^{8} .
\end{aligned}
$$

Any $c_{\mathrm{Z}}$ that comes from a solution of the system (7) must also be a root of $P$. However, using Maple, we found that the Galois group over $\mathbb{Q}\left(c_{\mathrm{A}}, c_{\mathrm{B}}, k, K\right)$ of the equation $P\left(c_{\mathrm{Z}}\right)=0$ is $S_{8}$. Hence, according to Theorem $1, c_{\mathrm{Z}}$ cannot be found explicitly in terms of $c_{\mathrm{A}}$ and $c_{\mathrm{B}}$. Therefore, the QSSA equations are not solvable this case.

Remark 1. To simplify the computations, we set all reaction rates equal to $K, k$, or 1 in the above calculations. On the other hand, if it were possible to find explicit formulas using radicals for the general set of parameters $k_{1}, k_{-1}, k_{2}, k_{3}, k_{-3}, k_{4}, k_{5}$, then the same formulas would apply for the special case of the parameters $K, k, 1$, which, according to the computations above, is impossible.

The second example is a real chemical mechanism for the photochemical decomposition of propanone,

$$
\mathrm{CH}_{3} \mathrm{COCH}_{3} \longrightarrow \mathrm{C}_{2} \mathrm{H}_{6}+\mathrm{CO} \text {. }
$$

The following mechanism was proposed in [25]:

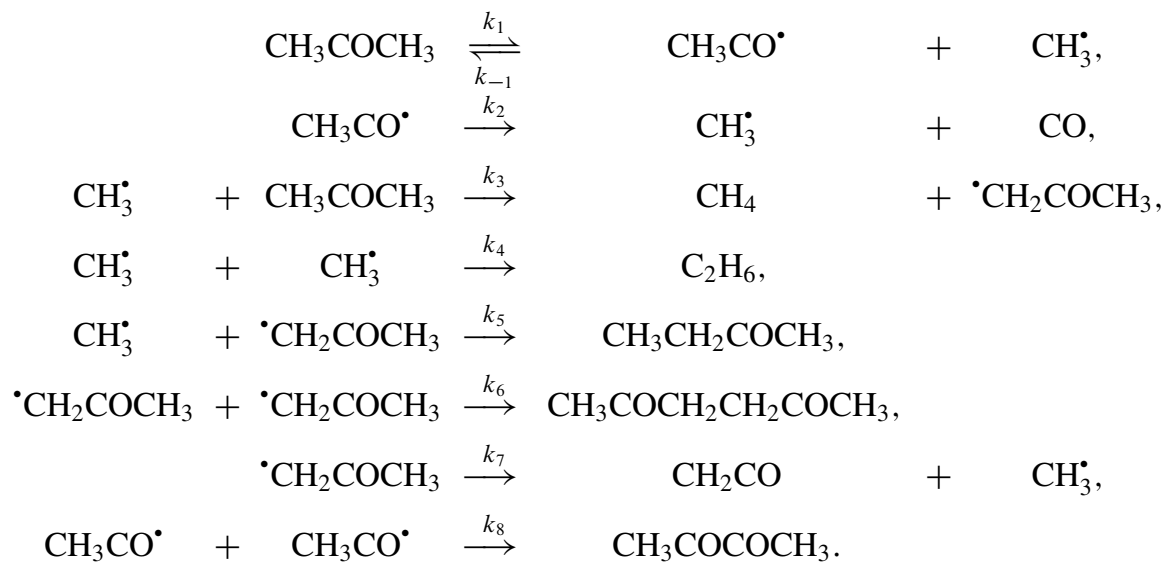


To simplify the notation, we write

$$
\begin{gathered}
\mathrm{A}=\mathrm{CH}_{3} \mathrm{COCH}_{3}, \mathrm{~B}=\mathrm{CO}, \mathrm{C}=\mathrm{C}_{2} \mathrm{H}_{6}, \mathrm{D}=\mathrm{CH}_{4}, \mathrm{E}=\mathrm{CH}_{3} \mathrm{CH}_{2} \mathrm{COCH}_{3}, \\
\mathrm{~F}=\mathrm{CH}_{3} \mathrm{COCH}_{2} \mathrm{CH}_{2} \mathrm{COCH}_{3}, \mathrm{G}=\mathrm{CH}_{2} \mathrm{CO}, \\
\mathrm{H}=\mathrm{CH}_{3} \mathrm{COCOCH}_{3}, \mathrm{X}=\mathrm{CH}_{3} \mathrm{CO}, \mathrm{Y}=\mathrm{CH}_{3}^{\bullet}, \mathrm{Z}={ }^{\circ} \mathrm{CH}_{2} \mathrm{COCH}_{3} .
\end{gathered}
$$

The corresponding system of differential equations is

$$
\begin{aligned}
& \dot{c}_{\mathrm{A}}=-k_{1} c_{\mathrm{A}}+k_{-1} c_{\mathrm{X}} c_{\mathrm{Y}}-k_{3} c_{\mathrm{A}} c_{\mathrm{Y}}, \\
& \dot{c}_{\mathrm{B}}=k_{2} c_{\mathrm{X}}, \\
& \dot{c}_{\mathrm{C}}=k_{4} c_{\mathrm{Y}}^{2}, \\
& \dot{c}_{\mathrm{D}}=k_{3} c_{\mathrm{A}} c_{\mathrm{Y}} \\
& \dot{c}_{\mathrm{E}}=k_{5} c_{\mathrm{Y}} c_{\mathrm{Z}} \\
& \dot{c}_{\mathrm{F}}=k_{6} c_{\mathrm{Z}}^{2} \\
& \dot{c}_{\mathrm{G}}=k_{7} c_{\mathrm{Z}}, \\
& \dot{c}_{\mathrm{H}}=k_{8} c_{\mathrm{X}}^{2}, \\
& \dot{c}_{\mathrm{X}}=k_{1} c_{\mathrm{A}}-k_{-1} c_{\mathrm{X}} c_{\mathrm{Y}}-k_{2} c_{\mathrm{X}}-2 k_{8} c_{\mathrm{X}}^{2}, \\
& \dot{c}_{\mathrm{Y}}=k_{1} c_{\mathrm{A}}+k_{2} c_{\mathrm{X}}-k_{-1} c_{\mathrm{X}} c_{\mathrm{Y}}-k_{3} c_{\mathrm{A}} c_{\mathrm{Y}}-2 k_{4} c_{\mathrm{Y}}^{2}-k_{5} c_{\mathrm{Y}} c_{\mathrm{Z}}+k_{7} c_{\mathrm{Z}}, \\
& \dot{c}_{\mathrm{Z}}=k_{3} c_{\mathrm{A}} c_{\mathrm{Y}}-k_{5} c_{\mathrm{Y}} c_{\mathrm{Z}}-2 k_{6} c_{\mathrm{Z}}^{2}-k_{7} c_{\mathrm{Z}} .
\end{aligned}
$$

The prospective QSSA intermediates are the radicals $\mathrm{X}, \mathrm{Y}$, and $\mathrm{Z}$. The algebraic system in $c_{\mathrm{X}}, c_{\mathrm{Y}}$, and $c_{\mathrm{Z}}$ is

$$
\begin{array}{r}
k_{1} c_{\mathrm{A}}-k_{-1} c_{\mathrm{X}} c_{\mathrm{Y}}-k_{2} c_{\mathrm{X}}-2 k_{8} c_{\mathrm{X}}^{2}=0, \\
k_{1} c_{\mathrm{A}}+k_{2} c_{\mathrm{X}}-k_{-1} c_{\mathrm{X}} c_{\mathrm{Y}}-k_{3} c_{\mathrm{A}} c_{\mathrm{Y}}-2 k_{4} c_{\mathrm{Y}}^{2}-k_{5} c_{\mathrm{Y}} c_{\mathrm{Z}}+k_{7} c_{\mathrm{Z}}=0, \\
k_{3} c_{\mathrm{A}} c_{\mathrm{Y}}-k_{5} c_{\mathrm{Y}} c_{\mathrm{Z}}-2 k_{6} c_{\mathrm{Z}}^{2}-k_{7} c_{\mathrm{Z}}=0 .
\end{array}
$$

If we choose all reaction rate parameters to be 1 , then this system has a Groebner basis that contains the following univariate polynomial in $c_{\mathrm{Z}}$ :

$$
\begin{aligned}
R\left(c_{\mathrm{Z}}\right)= & -c_{\mathrm{A}}^{5}+c_{\mathrm{A}}^{6}+\left(5 c_{\mathrm{A}}^{4}-4 c_{\mathrm{A}}^{5}\right) c_{\mathrm{Z}}+\left(-12 c_{\mathrm{A}}^{3}-4 c_{\mathrm{A}}^{5}+c_{\mathrm{A}}^{2}+5 c_{\mathrm{A}}^{4}\right) c_{\mathrm{Z}}^{2}+ \\
& \left(-16 c_{\mathrm{A}}^{3}+16 c_{\mathrm{A}}^{2}-2 c_{\mathrm{A}}+8 c_{\mathrm{A}}^{4}\right) c_{\mathrm{Z}}^{3}+\left(4+42 c_{\mathrm{A}}^{2}-4 c_{\mathrm{A}}-8 c_{\mathrm{A}}^{3}+4 c_{\mathrm{A}}^{4}\right) c_{\mathrm{Z}}^{4}+ \\
& \left(44 c_{\mathrm{A}}^{2}+20\right) c_{\mathrm{Z}}^{5}+\left(44+8 c_{\mathrm{A}}+20 c_{\mathrm{A}}^{2}\right) c_{\mathrm{Z}}^{6}+48 c_{\mathrm{Z}}^{7}+24 c_{\mathrm{Z}}^{8} .
\end{aligned}
$$


As in the previous example, the Galois group of the polynomial $R\left(c_{Z}\right)$ over the field $\mathbb{Q}\left(c_{\mathrm{A}}\right)$ is $S_{8}$. Therefore $c_{\mathrm{X}}, c_{\mathrm{Y}}$, and $c_{\mathrm{Z}}$ cannot be expressed in terms of $c_{\mathrm{A}}$, i.e., the QSSA approach is not solvable in this case.

\subsection{Challenges in the Characterization of Systems to Which the Standard QSSA Procedure Can Be Applied}

The method described in the previous section can be extended to an algorithm for checking whether the standard QSSA procedure can be applied to a generic system. With all rate constants regarded as symbolic entries, Groebner bases are computed using appropriate monomial orderings, and univariate polynomials are obtained for each intermediate. If all corresponding Galois groups are solvable, we conclude that the standard QSSA procedure may be applied. If at least one such group is not solvable, then the standard QSSA procedure cannot be carried out.

On the other hand, calculations of Groebner bases are combinatorially explosive even for polynomials over real numbers, and are even more computationally expensive over fraction fields with several variables. Therefore, for large enough networks, the computation may not terminate. Moreover, even if the computation terminates and the standard QSSA procedure can be applied, constructing the actual equations of the reduced model may involve further complications, including convoluted algebraic expressions whose signs need to be analyzed, and the possibility of multiple nonnegative roots of the QSSA algebraic system.

Setting some rate constants to simple numerical values, as we have done above, can reduce dramatically the computational size of the problem. However, while this easier problem may be computationally tractable, it is only informative if one of the resulting Galois groups is not solvable. In that case we can conclude that the QSSA procedure cannot be applied to the system with general reaction rates. On the other hand, even if all resulting Galois groups are solvable for some fixed reaction rate values, we cannot conclude that the system with general reaction rates is solvable.

\section{Alternatives to Solving the QSSA Equations}

\subsection{Eliminating Reactions Involving Intermediates}

The goal in QSSA model reduction is usually not to evaluate the concentrations of the intermediates but to eliminate them from the reduced model. We next investigate whether there is a general method to remove them short of solving for them. Consider first an example in which this elimination can be done:

$$
\mathrm{A} \stackrel{r_{1}}{\longrightarrow} \mathrm{B}, \quad \mathrm{B} \stackrel{r_{2}}{\longrightarrow} \mathrm{C} .
$$


We do not assume any simple form for the rate expressions. If $\mathrm{B}$ is an intermediate, we set its production rate to zero and obtain

$$
R_{\mathrm{B}}=r_{1}\left(c_{\mathrm{A}}\right)-r_{2}\left(c_{\mathrm{B}}\right)=0
$$

This relationship defines $c_{\mathrm{B}}$ as an implicit function of $c_{\mathrm{A}}$, but we assume that the algebraic complexity of the function $r_{2}$ prevents solving this equation explicitly. For this simple example, knowing only that $r_{2}\left(c_{\mathrm{B}}\right)=r_{1}\left(c_{\mathrm{A}}\right)$ is sufficient. In fact, the production rates of $\mathrm{A}$ and $\mathrm{C}$ using $r_{2}=r_{1}$ are expressible by

$$
R_{\mathrm{A}}=-r_{1}(\mathrm{~A}), \quad R_{\mathrm{C}}=r_{1}(\mathrm{~A}),
$$

and we have eliminated $\mathrm{B}$ and any large rate constants from the model without solving explicitly for $c_{\mathrm{B}}$ as a function of $c_{\mathrm{A}}$, i.e., $c_{\mathrm{B}}\left(c_{\mathrm{A}}\right)$.

But now consider a slightly more complex example:

$$
\mathrm{A} \stackrel{r_{1}}{\longrightarrow} 2 \mathrm{~B}, \quad 2 \mathrm{~B} \stackrel{r_{2}}{\longrightarrow} \mathrm{D}, \quad \mathrm{B}+\mathrm{C} \stackrel{r_{3}}{\longrightarrow} \mathrm{E} .
$$

If we say $B$ is an intermediate again, we have

$$
R_{\mathrm{B}}=2 r_{1}\left(c_{\mathrm{A}}\right)-2 r_{2}\left(c_{\mathrm{B}}\right)-r_{3}\left(c_{\mathrm{B}}, c_{\mathrm{C}}\right)=0
$$

In general, this equation implicitly defines a function $c_{\mathrm{B}}\left(c_{\mathrm{A}}, c_{\mathrm{C}}\right)$, but we again assume that we cannot solve for this function explicitly. The production rates of the reactants and products are as follows:

$$
R_{\mathrm{A}}=-r_{1}\left(c_{\mathrm{A}}\right), \quad R_{\mathrm{C}}=-r_{3}\left(c_{\mathrm{B}}, c_{\mathrm{C}}\right), \quad R_{\mathrm{D}}=r_{2}\left(c_{\mathrm{B}}\right), \quad R_{\mathrm{E}}=r_{3}\left(c_{\mathrm{B}}, c_{\mathrm{C}}\right)
$$

To eliminate B from the model, we require $r_{2}$ and $r_{3}$. But the QSSA relation provides only the relation $2 r_{1}-2 r_{2}-r_{3}=0$. We can remove $r_{2}$ or $r_{3}$ from the model using this equation, but not both. We require the function $c_{\mathrm{B}}\left(c_{\mathrm{A}}, c_{\mathrm{C}}\right)$ to substitute into $r_{2}$ and $r_{3}$ in order to remove $\mathrm{B}$ from the reduced model in this example. Eliminating reactions is not a general procedure. If the number of reaction rate expressions in which intermediates appear exceeds the number of intermediates, we do not obtain enough equations to eliminate the intermediates. In the preceding example, we have one intermediate (B), but it appears in two reaction rate expressions $\left(r_{2}, r_{3}\right)$.

The generalization of this idea of elimination to sets of reactions is known as the Horiuti-Temkin theory $[13,22]$. We shall not discuss this generalization further here, because the second simple example shows that the Horiuti-Temkin theory is insufficient in general to eliminate the reaction rates containing intermediates as required to apply the QSSA. 


\subsection{Solving the Full or Reduced Model Numerically}

Since we cannot solve the algebra of the QSSA and we cannot eliminate all reaction rates containing intermediates, we consider next the numerical solution of the full model. A simple approach is to set the rate constants corresponding to consumption of the intermediates large and solve the full model. We assume that the ODE solver can provide accurate solutions given any choice of large rate constants. Alternatively, one can replace the differential equations for the intermediates with the algebraic equations that result from setting their production rates to zero. This procedure produces a set of differential-algebraic equations (DAEs) in place of the ODEs of the full model. The procedure for generating and numerically solving these DAE models is known as computational singular perturbation (CSP). A discussion of the CSP method is provided in $[16,26,27]$. Whether one is numerically solving the full model or the reduced, slow-timescale DAE model, it is generally assumed that all kinetic parameters are known.

To illustrate the issues that arise when the large rate constants are not all known, we consider the following example:

$$
\mathrm{A} \stackrel{k_{1}}{\longrightarrow} \mathrm{B}, \quad \mathrm{B} \stackrel{k_{2}}{\longrightarrow} \mathrm{C}, \quad 2 \mathrm{~B} \stackrel{k_{3}}{\longrightarrow} \mathrm{D}
$$

with the full model

$$
\begin{aligned}
& \dot{c}_{\mathrm{A}}=-k_{1} c_{\mathrm{A}}, \\
& \dot{c}_{\mathrm{B}}=k_{1} c_{\mathrm{A}}-k_{2} c_{\mathrm{B}}-2 k_{3} c_{\mathrm{B}}^{2}, \\
& \dot{c}_{\mathrm{C}}=k_{2} c_{\mathrm{B}}, \\
& \dot{c}_{\mathrm{D}}=k_{3} c_{\mathrm{B}}^{2} .
\end{aligned}
$$

The production rate of $\mathrm{B}$ is given by

$$
R_{\mathrm{B}}=k_{1} c_{\mathrm{A}}-k_{2} c_{\mathrm{B}}-2 k_{3} c_{\mathrm{B}}^{2} .
$$

Setting this production rate to zero and solving for $c_{\mathrm{B}}$ gives the QSSA result,

$$
c_{\mathrm{B}}=\frac{2 k_{1} / k_{2}}{1+\sqrt{1+\beta c_{\mathrm{A}}}} c_{\mathrm{A}}, \quad \beta=8 k_{1} k_{3} / k_{2}^{2} .
$$

We then express the production rates in terms of the concentrations of only the reactants and products $(\mathrm{A}, \mathrm{C}$, and $\mathrm{D})$

$$
\begin{aligned}
& R_{\mathrm{A}}=-k_{1} c_{\mathrm{A}}, \\
& R_{\mathrm{C}}=\frac{2 k_{1}}{1+\sqrt{1+\beta c_{\mathrm{A}}}} c_{\mathrm{A}},
\end{aligned}
$$


a
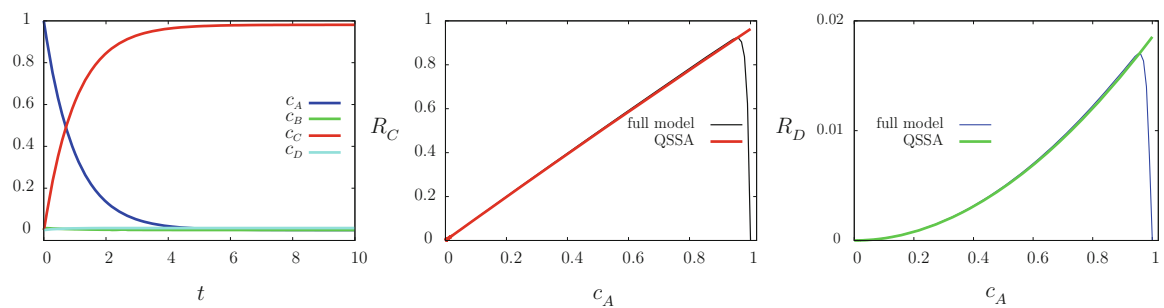

b
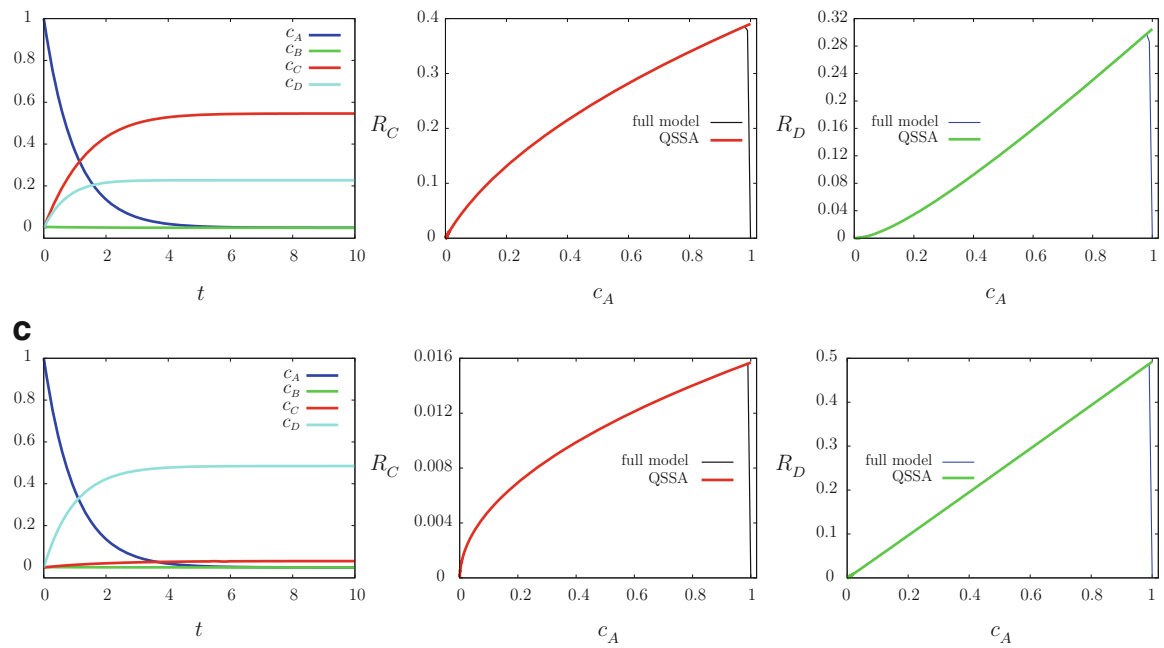

Fig. 2 Numerical solutions of the full model and QSSA approximation. Left: $c_{\mathrm{A}}, c_{\mathrm{B}}, c_{\mathrm{C}}, c_{\mathrm{D}}$ versus time. Middle: $R_{\mathrm{C}}$ versus $c_{\mathrm{A}}$. Right: $R_{\mathrm{D}}$ versus $c_{\mathrm{A}}$. (a) $k_{1}=1, k_{2}=10^{2}, k_{3}=2 \times 10^{2}, \beta=$ $16 \times 10^{-2} \cdot R_{\mathrm{C}}$ versus $c_{\mathrm{A}}$ is linear. (b) $k_{1}=1, k_{2}=10^{2}, k_{3}=2 \times 10^{4}, \beta=16$. Neither $R_{\mathrm{C}}$ nor $R_{\mathrm{D}}$ is linear with $c_{A}$. (c) $k_{1}=1, k_{2}=10, k_{3}=2 \times 10^{5}, \beta=16 \times 10^{3}$. $R_{\mathrm{D}}$ versus $c_{\mathrm{A}}$ is linear

$$
R_{\mathrm{D}}=\frac{1}{2} k_{1}\left(\frac{-1+\sqrt{1+\beta c_{\mathrm{A}}}}{1+\sqrt{1+\beta c_{\mathrm{A}}}}\right) c_{\mathrm{A}} \text {. }
$$

If we were unable to solve the algebra, and instead tried the simple approach of setting all rate constants for consumption of intermediates large, we would set $k_{2}$ and $k_{3}$ large. But this does not specify the value of $\beta$. If we examine the two limiting cases of $\beta$ large and $\beta$ small, we obtain the production rates

$$
\begin{aligned}
\beta & \rightarrow 0, & \beta & \rightarrow \infty, \\
R_{\mathrm{A}} & =-k_{1} c_{\mathrm{A}}, & R_{\mathrm{A}} & =-k_{1} c_{\mathrm{A}}, \\
R_{\mathrm{C}} & =k_{1} c_{\mathrm{A}}, & & R_{\mathrm{C}}=0,
\end{aligned}
$$




$$
R_{\mathrm{D}}=0, \quad R_{\mathrm{D}}=\frac{1}{2} k_{1} c_{\mathrm{A}}
$$

corresponding to the two reduced mechanisms

$$
\mathrm{A} \stackrel{k_{1}}{\longrightarrow} \mathrm{C} \quad(\beta \rightarrow 0), \quad \mathrm{A} \stackrel{k_{1}}{\longrightarrow} \frac{1}{2} \mathrm{D} \quad(\beta \rightarrow \infty) .
$$

Some relevant numerical results for the example (10) are shown in Fig. 2. Notice that neither the limit $k_{2} \gg k_{3}$ nor $k_{3} \gg k_{2}$ can describe the behavior of the QSSA model for the intermediate range of $\beta$ shown in Fig. 2b. We see that the QSSA treatment of the full model remains valid, but we cannot obtain the correct behavior by simply setting rate constants large. We need to know the relative sizes of some large rate constants; in this case, we need $k_{3} / k_{2}^{2}$. It is a simple matter to estimate the parameters $k_{1}$ and $\beta$ from measurements of $\mathrm{C}$ and $\mathrm{D}$, but it is not a simple matter to know the form of the production rates of $C$ and $D$ given in Eqs. (13) and (14). The knowledge of the form of the production rates is the primary benefit of the standard QSSA in this simple example.

\section{Rescaling Intermediates, and Parameter Estimation from Data}

Since solving for the intermediates in closed form is not always possible, in this section we explore an alternative procedure of rescaling the intermediates. The choice of how to rescale is not unique, but we show in the next section how to automate the procedure for any kinetic model; a primary benefit is that the rescaling procedure often provides a tractable parameter estimation problem as well. For illustrative purposes, consider again the previous example, but assume that we are unable to solve the algebra to determine $c_{\mathrm{B}}$ from setting $R_{\mathrm{B}}=0$. Instead, we introduce a rescaled $\mathrm{B}$ concentration, denoted $c_{\mathrm{Z}}$, by writing

$$
c_{\mathrm{Z}}=k_{2} c_{\mathrm{B}} .
$$

Note that this choice is not unique. Setting $R_{\mathrm{B}}=0$ then adds an algebraic equation to the other species' differential equations. The reduced DAE model is

$$
\begin{aligned}
\dot{c}_{\mathrm{A}} & =-k_{1} c_{\mathrm{A}}, \\
0 & =k_{1} c_{\mathrm{A}}-c_{\mathrm{Z}}-2 K_{3} c_{\mathrm{Z}}^{2}, \\
\dot{c}_{\mathrm{C}} & =c_{\mathrm{Z}}, \\
\dot{c}_{\mathrm{D}} & =K_{3} c_{\mathrm{Z}}^{2} .
\end{aligned}
$$


Fig. 3 Measurement of the concentration of $\mathrm{C}$ versus time and prediction of the concentrations of $\mathrm{A}, \mathrm{B}$, and $\mathrm{C}$ from the rescaled model using optimal parameter estimates

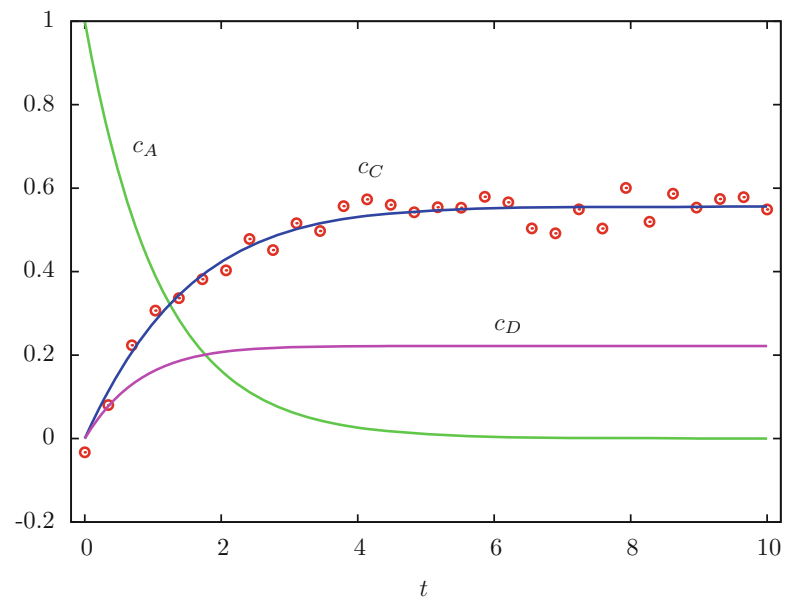

Notice that the rescaling removes the two potentially large kinetic parameters $k_{2}$ and $k_{3}$ and introduces one new scaled parameter, $K_{3}=k_{3} / k_{2}^{2}$. Without concentration measurements of reactants and products, we have no idea about the size of $K_{3}$; the low concentration of the intermediate $\mathrm{B}$ tells us only that $k_{3}$ or $k_{2}$ or both are large, and it is silent regarding the ratio $k_{3} / k_{2}^{2}$.

Next, we attempt parameter estimation using both the full and the reduced models. Consider the data set with measurement noise depicted in Fig. 3. To make the parameter estimation problem challenging, we assume that only species $\mathrm{C}$ can be measured conveniently at the fairly slow sampling rate $(\Delta=0.34)$ shown in the figure. This data set was generated from the full model using $k_{1}=1.0, k_{2}=100$, and $k_{3}=2 \times 10^{4}$, and the initial conditions $c_{\mathrm{A} 0}=1.0$ and $c_{\mathrm{B} 0}=c_{\mathrm{C} 0}=c_{\mathrm{D} 0}=0.0$. Note that these choices correspond to the intermediate case (b) in Fig. 2.

Normally distributed measurement noise (zero mean, standard deviation $=0.03$ ) was added to the $c_{\mathrm{C}}$ of the full model to create the measurement set. Attempting to estimate $k_{1}, k_{2}, k_{3}$ of the full model from these data is hopeless. An optimizer would find a set of infinitely many estimates with approximately constant $k_{2} / k_{3}^{2}$, and all points in this set would fit the measurements equally well. This lack of identifiability of parameters plagues all overly complex models using realistic measurement sets.

The estimates obtained for the parameters $k_{1}, K_{3}$ of the reduced model are shown in Fig. 4. Notice that the parameters are well determined and that the approximate $95 \%$ confidence interval is small and contains the "true" parameters used to generate the data. ${ }^{1}$ The method used to generate the confidence interval is discussed in standard texts $[3,19]$. The values of the estimates and the plus/minus interval (the bounding box of the ellipse shown in Fig. 4) are

\footnotetext{
${ }^{1}$ Note that these are not quite the true parameter values, because we used the full model rather than the reduced model to generate the data.
} 


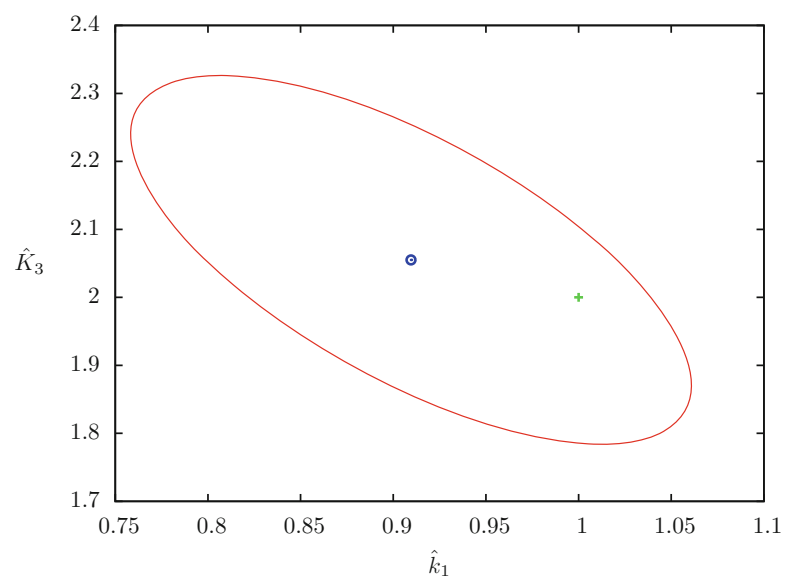

Fig. 4 Optimal parameter estimates $\hat{k}_{1}$ and $\hat{K}_{3}(\odot)$ based on the measurements of species $\mathrm{C}$ in Fig. 3, approximate $95 \%$ confidence interval (line), and parameter values used to create the measurements (+)

$$
\left[\begin{array}{l}
\hat{k}_{1} \\
\hat{K}_{3}
\end{array}\right]=\left[\begin{array}{c}
0.910 \\
2.06
\end{array}\right] \pm\left[\begin{array}{l}
0.151 \\
0.271
\end{array}\right], \quad\left[\begin{array}{c}
k_{1} \\
k_{3} \\
k_{2}^{2}
\end{array}\right]=\left[\begin{array}{l}
1.0 \\
2.0
\end{array}\right] .
$$

As we can see, applying the QSSA to the full model provides a validated reduced model that is useful for other scientific studies and for engineering design purposes.

\section{A Reparametrization of the QSSA Model}

Recall the rescaling of intermediates presented in the previous section, where two kinetic parameters $k_{2}$ and $k_{3}$ were replaced by a single new parameter $K_{3}$. In this section, we show how this rescaling procedure can be performed for any set of QSSA differential-algebraic equations, and highlight some interesting properties of the rescaled model.

Let $(\mathcal{S})$ denote a system of DAEs corresponding to a QSSA model with unknown kinetic parameters and with variables $x_{1}, \ldots, x_{n}$. Suppose that the first $m$ variables, $x_{1}, \ldots, x_{m}$, correspond to nonintermediate chemical species, and that the variables $x_{m+1}, \ldots, x_{n}$ correspond to intermediate species. We assume that $(\mathcal{S})$ fulfills the following assumption, which is satisfied by most QSSA systems encountered in practice: for any $t \geq 0$, if $x_{1}(t), \ldots, x_{m}(t)$ are known, then the $n-m+1$ algebraic equations of $(\mathcal{S})$ have unique nonnegative solutions for $x_{m+1}(t), \ldots, x_{n}(t)$ (note that, as illustrated in Sect. 2.3, these solutions may not be expressible by radicals). As a consequence, a solution of $(\mathcal{S})$ is determined by specifying only the first $m$ coordinates of the initial condition vector. 
In what follows, we devise a simpler model $(\overline{\mathcal{S}})$ of $(\mathcal{S})$ with variables $\bar{x}_{1}, \ldots, \bar{x}_{n}$ that captures the dynamics of the nonintermediate species $x_{1}, \ldots, x_{m}$. More precisely, $(\overline{\mathcal{S}})$ will satisfy the following two properties:

(i) $(\overline{\mathcal{S}})$ has fewer parameters than $(\mathcal{S})$;

(ii) If $\left(x_{1}(t), \ldots, x_{n}(t)\right)$ and $\left(\bar{x}_{1}(t), \ldots, \bar{x}_{n}(t)\right), t \geq 0$, are solutions of $(\mathcal{S})$ and $(\overline{\mathcal{S}})$ with $x_{i}(0)=\bar{x}_{i}(0)$ for all $i \in\{1, \ldots, m\}$, then $x_{i}(t)=\bar{x}_{i}(t)$ for all $i \in\{1, \ldots, m\}$ and all $t \geq 0$.

In other words, condition (ii) specifies that the outputs $x_{1}, \ldots, x_{m}$ from $(\overline{\mathcal{S}})$ and $(\mathcal{S})$ corresponding to nonintermediates are the same.

\subsection{Rescaling of the Intermediates}

Our strategy is to consider rescalings of intermediates

$$
\bar{x}_{i}=\alpha_{i} x_{i} \text { for } i \in\{m+1, \ldots, n\}
$$

that are "optimal" in the sense that the rescaled DAE system has the minimum number of parameters that can be obtained by any reparametrization of the form (16). More precisely, the $\alpha_{i}$ are chosen such that some monomials with unknown coefficients in $(\mathcal{S})$ are replaced by monomials with coefficient 1 in the reparametrized system $(\overline{\mathcal{S}})$, and this is achieved for as many monomials as possible.

To illustrate, suppose that the DAE system $(\mathcal{S})$ has intermediate variables $x, y, z, w, u$ and suppose that the monomials of $(\mathcal{S})$ involving the intermediate variables are

$$
k_{1} x y, k_{2} z w, k_{3} y z, k_{4} x w, k_{5} w u \text {, and } k_{6} y u \text {. }
$$

Note that these monomials might contain more factors corresponding to nonintermediate variables, but, since these factors do not play a role in our analysis, we will neglect them.

Since the kinetic parameters $k_{1}, \ldots, k_{6}$ are unknown positive constants, we treat them as (linearly independent) symbolic indeterminates. Letting

$$
\bar{x}=\alpha x, \bar{y}=\beta y, \bar{z}=\gamma z, \bar{w}=\delta w, \bar{u}=\eta u,
$$

the monomials become

$$
\frac{k_{1}}{\alpha \beta} \bar{x} \bar{y}, \frac{k_{2}}{\gamma \delta} \bar{z} \bar{w}, \frac{k_{3}}{\beta \gamma} \bar{y} \bar{z}, \frac{k_{4}}{\alpha \delta} \bar{x} \bar{w}, \frac{k_{5}}{\delta \eta} \bar{w} \bar{u}, \frac{k_{6}}{\beta \eta} \bar{y} \bar{u} .
$$


Let $\mathbf{C}$ denote the vector of the logarithms of the six coefficients in the monomials above, and let $\ln \mathbf{k}, \mathbf{v}$, and $\Psi$ denote the $6 \times 1$ and $5 \times 1$ vectors and the $6 \times 5$ matrix, respectively, in the equality

$$
\mathbf{C}=\ln \mathbf{k}-\Psi \mathbf{v}=\left[\begin{array}{l}
\ln k_{1} \\
\ln k_{2} \\
\ln k_{3} \\
\ln k_{4} \\
\ln k_{5} \\
\ln k_{6}
\end{array}\right]-\left[\begin{array}{lllll}
1 & 1 & 0 & 0 & 0 \\
0 & 0 & 1 & 1 & 0 \\
0 & 1 & 1 & 0 & 0 \\
1 & 0 & 0 & 1 & 0 \\
0 & 0 & 0 & 1 & 1 \\
0 & 1 & 0 & 0 & 1
\end{array}\right]\left[\begin{array}{l}
\ln \alpha \\
\ln \beta \\
\ln \gamma \\
\ln \delta \\
\ln \eta
\end{array}\right]=\left[\begin{array}{l}
\ln k_{1} \\
\ln k_{2} \\
\ln k_{3} \\
\ln k_{4} \\
\ln k_{5} \\
\ln k_{6}
\end{array}\right]-\left[\begin{array}{c}
M_{1} \cdot \mathbf{v} \\
M_{2} \cdot \mathbf{v} \\
M_{3} \cdot \mathbf{v} \\
M_{4} \cdot \mathbf{v} \\
M_{5} \cdot \mathbf{v} \\
M_{6} \cdot \mathbf{v}
\end{array}\right],
$$

where $M_{j}$ denotes row $j$ of $\Psi$. The maximum number of zero coordinates for the vector $\mathbf{C}$ equals $\operatorname{rank} \Psi=4$.

Since $M_{1}, M_{2}, M_{3}$, and $M_{5}$ are linearly independent, we can make the corresponding coordinates of $\mathbf{C}$ equal to zero (and therefore make the coefficients of the first two monomials in (17) equal to 1 ). We have

$$
\mathbf{C}=\left[\begin{array}{l}
\ln k_{1} \\
\ln k_{2} \\
\ln k_{3} \\
\ln k_{4} \\
\ln k_{5} \\
\ln k_{6}
\end{array}\right]-\left[\begin{array}{c}
M_{1} \cdot \mathbf{v} \\
M_{2} \cdot \mathbf{v} \\
M_{3} \cdot \mathbf{v} \\
\left(M_{1}+M_{2}-M_{3}\right) \cdot \mathbf{v} \\
M_{5} \cdot \mathbf{v} \\
\left(M_{1}+M_{5}-M_{4}\right) \cdot \mathbf{v}
\end{array}\right]=\left[\begin{array}{l}
\ln k_{1} \\
\ln k_{2} \\
\ln k_{3} \\
\ln k_{4} \\
\ln k_{5} \\
\ln k_{6}
\end{array}\right]-\left[\begin{array}{c}
\ln k_{1} \\
\ln k_{2} \\
\ln k_{3} \\
\ln k_{1}+\ln k_{2}-\ln k_{3} \\
\ln k_{5} \\
\ln k_{1}+\ln k_{5}-\ln k_{4}
\end{array}\right] .
$$

The new monomials

$$
\bar{x} \bar{y}, \bar{z} \bar{w}, \bar{y} \bar{z}, K_{1} \bar{x} \bar{w}, \bar{w} \bar{u} \text {, and } K_{2} \bar{y} \bar{u}
$$

contain only two parameters,

$$
K_{1}=\frac{k_{4} k_{3}}{k_{1} k_{2}} \text { and } K_{2}=\frac{k_{6} k_{4}}{k_{1} k_{5}} .
$$

In general, the number of new parameters is equal to the number of old parameters minus the rank of $\Psi$.

\section{Remarks.}

1. The reparametrized system $(\mathcal{S})$ indeed satisfies the desired properties $(i)$ and (ii). Condition $(i)$ is true, as explained above. Also, the differential equations for $\bar{x}_{1}, \ldots, \bar{x}_{m}$ in $(\overline{\mathcal{S}})$ are exactly the same as the corresponding differential equations for $x_{1}, \ldots, x_{m}$ in $(\mathcal{S})$; therefore, for identical initial conditions, the solutions must coincide, i.e., $\bar{x}_{i}=x_{i}$ for $i \in\{1, \ldots, m\}$. 
2. Our example shows that the optimal scaling is not always obvious, and that, in general, the number of parameters that can be eliminated may be smaller than the number of intermediates present in the system.

3. Note that, in some cases, even after reducing the number of parameters as described above, we might not obtain a system with uniquely identifiable parameters. This may be the case even if there are no intermediate species; see [8] for examples. In future work, we will analyze this issue in more detail.

\subsection{Equivalence of Reparametrizations}

As explained above, the reparametrization (19) is optimal with respect to the number of parameters that it removes. However, this reparametrization is not the only one that is optimal. Similarly to the way $M_{1}, M_{2}, M_{3}$, and $M_{5}$ determined an optimal reparametrization in the previous section, any choice of linearly independent rows of $\Psi$ determines another optimal reparametrization.

Recall that the new parameters $K_{1}$ and $K_{2}$ in (19) are products of powers (positive or negative) of the original parameters $k_{1}, \ldots, k_{6}$. This is true for any reparametrization. Interestingly, any two optimal reparametrizations of a system $(\mathcal{S})$, with parameter sets denoted $\mathcal{P}$ and $\tilde{\mathcal{P}}$, are parameter-set equivalent, i.e., any element of $\tilde{\mathcal{P}}$ is a product of powers of elements of $\mathcal{P}$ and vice versa.

For example, if we choose $\mathbf{v}$ in (18) such that the second, fourth, fifth, and sixth coordinates of $\mathbf{C}$ are zero (using the fact that $M_{2}, M_{4}, M_{5}$, and $M_{6}$ are linearly independent), we have

$$
\mathbf{C}=\left[\begin{array}{l}
\ln k_{1} \\
\ln k_{2} \\
\ln k_{3} \\
\ln k_{4} \\
\ln k_{5} \\
\ln k_{6}
\end{array}\right]-\left[\begin{array}{c}
\left(M_{4}+M_{6}-M_{5}\right) \cdot \mathbf{v} \\
M_{2} \cdot \mathbf{v} \\
\left(M_{2}+M_{6}-M_{5}\right) \cdot \mathbf{v} \\
M_{4} \cdot \mathbf{v} \\
M_{5} \cdot \mathbf{v} \\
M_{6} \cdot \mathbf{v}
\end{array}\right]=\left[\begin{array}{l}
\ln k_{1} \\
\ln k_{2} \\
\ln k_{3} \\
\ln k_{4} \\
\ln k_{5} \\
\ln k_{6}
\end{array}\right]-\left[\begin{array}{c}
\ln k_{4}+\ln k_{6}-\ln k_{5} \\
\ln k_{2} \\
\ln k_{2}+\ln k_{6}-\ln k_{5} \\
\ln k_{4} \\
\ln k_{5} \\
\ln k_{6}
\end{array}\right],
$$

and the new monomials are

$$
\tilde{K}_{1} \bar{x} \bar{y}, \tilde{K}_{2} \bar{z} \bar{w}, \bar{y} \bar{z}, \bar{x} \bar{w}, \bar{w} \bar{u} \text {, and } \bar{y} \bar{u},
$$

where

$$
\tilde{K}_{1}=\frac{k_{1} k_{5}}{k_{4} k_{6}} \text { and } \tilde{K}_{2}=\frac{k_{3} k_{5}}{k_{2} k_{6}} .
$$

Note that $\tilde{K}_{1}=1 / K_{2}$ and $\tilde{K}_{2}=K_{1} / K_{2}$, and the two reparametrizations are indeed parameter-set equivalent. 


\subsection{Propanone Example}

We shall now illustrate the usefulness of the reparametrization discussed in this section by estimating parameters for the photochemical decomposition of propanone. A representative data set was generated from the full model described by (8) using $k_{1}=10^{-2}, k_{-1}=10^{7}, k_{2}=10^{3}, k_{3}=10^{2}, k_{4}=5 \times 10^{4}, k_{5}=10^{3}, k_{6}=10^{1}$, $k_{7}=10^{-1}, k_{8}=10^{9}, c_{\mathrm{A} 0}=1, c_{\mathrm{B} 0}=c_{\mathrm{C} 0}=c_{\mathrm{D} 0}=c_{\mathrm{E} 0}=c_{\mathrm{F} 0}=c_{\mathrm{G} 0}=$ $c_{\mathrm{H} 0}=c_{\mathrm{X} 0}=c_{\mathrm{Y} 0}=c_{\mathrm{Z} 0}=0$. These parameter values were chosen so that the major products and reactants $\mathrm{A}, \mathrm{B}, \mathrm{C}, \mathrm{D}$, and $\mathrm{H}$ would be present in larger amounts than the minor species $\mathrm{E}, \mathrm{F}$, and $\mathrm{G}$, and the reactive intermediates $\mathrm{X}, \mathrm{Y}$, and $\mathrm{Z}$ would be present in much smaller quantities. Normally distributed measurement noise (zero mean, variance $=10^{-5}$ ) was added to $c_{\mathrm{A}}, c_{\mathrm{B}}, c_{\mathrm{C}}, c_{\mathrm{D}}, c_{\mathrm{E}}, c_{\mathrm{F}}, c_{\mathrm{G}}$, and $c_{\mathrm{H}}$ to create the measurement set. Similarly to the example represented by Eq. (10), estimating all nine parameters of the full model would result in infinitely many parameter sets which fit the data equally well. The reparametrized DAE model had six (rescaled) parameters $k_{1}, K_{-1}=k_{-1} /\left(k_{2} k_{3}\right), K_{4}=k_{4} / k_{3}^{2}, K_{5}=k_{5} /\left(k_{3} k_{7}\right)$, $K_{6}=k_{6} / k_{7}^{2}$, and $K_{8}=k_{8} / k_{2}^{2}$, which were estimated using the software package parest_dae to obtain a good fit to the measurement data and to obtain reasonably small $95 \%$ confidence intervals containing the "true" parameters used to create the measurement data. Figure 5 shows the fit between the measurement data and the estimated solution. The Estimated values of the rescaled parameters and their corresponding confidence intervals are

$$
\left[\begin{array}{c}
\hat{k}_{1} \\
\hat{K}_{-1} \\
\hat{K}_{4} \\
\hat{K}_{5} \\
\hat{K}_{6} \\
\hat{K}_{8}
\end{array}\right]=\left[\begin{array}{c}
0.0099 \\
98.4 \\
5.03 \\
101 \\
993 \\
996
\end{array}\right] \pm\left[\begin{array}{c}
0.0017 \\
82 \\
0.39 \\
5.03 \\
76 \\
68
\end{array}\right], \quad\left[\begin{array}{c}
k_{1} \\
K_{-1} \\
K_{4} \\
K_{5} \\
K_{6} \\
K_{8}
\end{array}\right]=\left[\begin{array}{c}
0.01 \\
100 \\
5 \\
100 \\
1000 \\
1000
\end{array}\right] .
$$

Note that the confidence interval for the parameter $\hat{K}_{-1}$ is the largest in a relative sense, indicating that this parameter is the least well determined by this experiment. If this uncertainty were deemed too large, one could then apply experimental design methods to determine the optimal experiment to be performed next in order to provide more information about this parameter [2,19]. This example shows, however, that although estimation of all of the parameters of the full model is not possible, reparametrization of the corresponding DAE model allows us to identify all of the parameters of the reparametrized DAE model. The reduced model is perfectly adequate for predicting the concentrations of all measurable species. If one were interested in identifying all of the full model's rate constants, different experiments using measurements of the QSSA species would be required. 

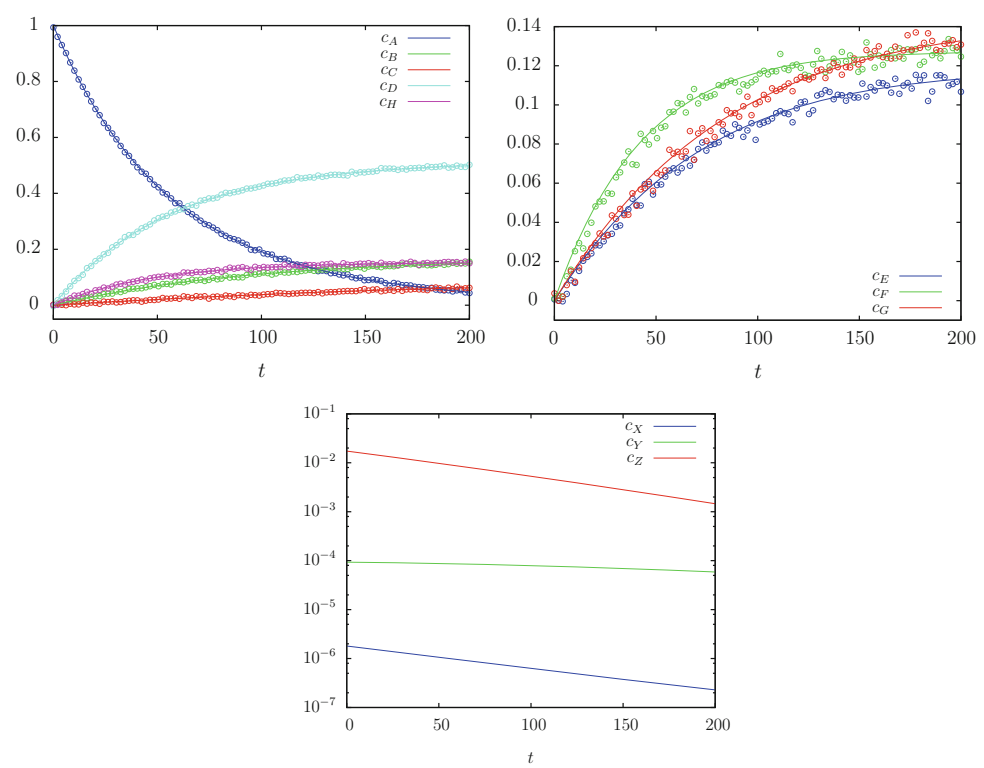

Fig. 5 Measurements of major and minor species and predictions of all species by the reduced DAE model. Top left: concentrations of major species $c_{\mathrm{A}}, c_{\mathrm{B}}, c_{\mathrm{C}}, c_{\mathrm{D}}, c_{\mathrm{H}}$ versus time. Top right: concentrations of minor species $c_{\mathrm{E}}, c_{\mathrm{F}}, c_{\mathrm{G}}$ versus time. Bottom: concentrations of reactive intermediates $c_{\mathrm{X}}, c_{\mathrm{Y}}, c_{\mathrm{Z}}$ versus time

\section{Conclusions}

The 100-year-old approach of classic QSSA model reduction, as taught in introductory courses, cannot be carried out for many relevant kinetics problems. We have proved that the algebra cannot be solved for even as few as five reactions involving five species (with three intermediates) with nothing more complex than bimolecular mass action kinetics. If any readers can find simpler nonsolvable examples, the authors would like to know about them. We have also analyzed a chemical mechanism taken from the literature and shown that it cannot be reduced by the classical approach. We have described algorithms that can test any mechanism for solvability. We have shown that the alternative approach of the Horiuti-Temkin theory also does not achieve the requirements of model reduction.

The first goal of this chapter was simply to make instructors aware of the limitations of trying to apply the QSSA in this way. Students should probably be told of these limitations when they are introduced to the approach. A second, longer-term goal was to promote the idea of rescaling the low-concentration species rather than solving for them. Although the choice of the rescaled parameters is not unique, the minimum number of rescaled parameters remaining in the reduced model $i s$ unique, and all reduced models with this minimum number of rescaled 
parameters are equivalent. We expect that in most large enough examples, one cannot solve the QSSA equations explicitly using radicals. Moreover, even when one can solve them explicitly, it may still be preferable to use rescaling in order to identify key combinations of parameters which can be identified from data. The result of the procedure can be a reduced (DAE) model with rescaled parameters that are identifiable from standard measurements. Calculations using the open source software package parest_dae that estimates parameters and confidence intervals for DAE models were presented. This package makes use of the recently released SUNDIALS implicit ODE solver IDAS [12].

Looking to the future, as ab initio methods for predicting rate constants become more capable, we may be able to reduce the number of large rate constants and functions of these large constants that must be estimated from measurements. Model validation studies may then be carried out numerically with the full model, rather than by inspecting the reduced model's structural dependence on large-concentration reactants and products. Further research and tool development supporting both rate constant prediction and numerical model validation should prove highly useful to scientists developing and using complex chemical models.

Acknowledgements We thank Andrei Căldăraru and Lev Borisov for useful suggestions and discussions. The work of CP and GC was partially supported by the DOE BACTER Institute, the National Science Foundation, and NIH grant R01GM86881.

\section{References}

1. N.H. Abel, Mémoire sur les équations algébriques, où l'on démontre l'impossibilité de la résolution de l'équation générale du cinquième degré in Oeuvres complètes de Niels Henrik Abel, Édition de Christiana, vol. 1 (Gröndahl and Son, Oslo, 1881), pp. 28-33

2. A.C. Atkinson, A.N. Donev, Optimum Experimental Designs (Oxford University Press, New York, 1992)

3. Y. Bard, Nonlinear Parameter Estimation (Academic, New York, 1974)

4. M. Bodenstein, Eine Theorie der photochemischen Reaktionsgeschwindigkeiten. Z. Phys. Chem. 85, 329-397 (1913)

5. D.L. Chapman, L.K. Underhill, The interaction of chlorine and hydrogen. The influence of mass. J. Chem. Soc. Trans. 103, 496-508 (1913)

6. A. Ciliberto, F. Capuani, J.J. Tyson, Modeling networks of coupled enzymatic reactions using the total quasi-steady state approximation. PLoS Comput. Biol. 3(3) e45 (2007)

7. D. Cox, J. Little, D. O'Shea, Ideals, Varieties, and Algorithms: An Introduction to Computational Algebraic Geometry and Commutative Algebra (Springer, New York, 2006)

8. G. Craciun, C. Pantea, Identifiability of chemical reaction networks. J. Math. Chem. 44, 244-259 (2007)

9. A. Goldbeter, D.E. Koshland, An amplified sensitivity arising from covalent modification in biological systems. Proc. Natl. Acad. Sci. U.S.A. 78(11), 6840-6844 (1981)

10. S. Hanson, S. Schnell, Reactant stationary approximation in enzyme kinetics. J. Phys. Chem. A 112(37), 8654-8658 (2008)

11. A.V. Hill, The possible effects of the aggregation of the molecules of hemoglobin on its dissociation curves. J. Physiol. 40, 4-7 (1910) 
12. A.C. Hindmarsh, P.N. Brown, K.E. Grant, S.L. Lee, R. Serban, D.E. Shumaker, C.S. Woodward, SUNDIALS: suite of nonlinear and differential/algebraic equation solvers. ACM Trans. Math. Softw. 31(3), 363-396 (2005)

13. J. Horiuti, T. Nakamura, Stoichiometric number and the theory of steady reaction. Z. Phys. Chem. 11, 358-365 (1957)

14. M. Isaacs, Algebra: A Graduate Course (Brooks Cole, Pacific Grove, 1993)

15. A.G. Khovanskii, On solvability and unsolvability of equations in explicit form. Russ. Math. Surv. 59(4), 661-736 (2004)

16. S.H. Lam, D.A. Goussis, The CSP method for simplifying kinetics. Int. J. Chem. Kinet. 26, 461-486 (1994)

17. Maple is a division of Waterloo Maple, Inc. (2007)

18. J.D. Murray, Mathematical Biology: I. An Introduction (Springer, New York, 2002)

19. J.B. Rawlings, J.G. Ekerdt, Chemical Reactor Analysis and Design Fundamentals (Nob Hill Publishing, Madison, 2004)

20. S. Schnell, P.K. Maini, A century of enzyme kinetics: reliability of the $K_{M}$ and $v_{\max }$ estimates. Comments Theor. Biol. 8, 169-187 (2003)

21. L.A. Segel, M. Slemrod, The quasi-steady state assumption: a case study in perturbation. SIAM Rev. 31(3), 446-477 (1989)

22. M.I. Temkin, The kinetics of some industrial heterogeneous catalytic reactions. Adv. Catal. 28, 173-291 (1979)

23. T. Turanyi, A.S. Tomlin, M.J. Pilling, On the error of the quasi-steady-state approximation. J. Phys. Chem. 97(1), 163-172 (1993)

24. J.J. Tyson, K.C. Chen, B. Novak, Sniffers, buzzers, toggles and blinkers: dynamics of regulatory and signaling pathways in the cell. Curr. Opin. Cell Biol. 15, 221-231 (2003)

25. M.R. Wright, An Introduction to Chemical Kinetics (Wiley, Chichester/Hoboken, 2004)

26. A. Zagaris, H.G. Kaper, T.J. Kaper, Analysis of the computational singular perturbation reduction method for chemical kinetics. J. Nonlinear Sci. 14, 59-91 (2004)

27. A. Zagaris, H.G. Kaper, T.J. Kaper, Fast and slow dynamics for the computational singular perturbation method. Multiscale Model. Simul. 2(4), 613-638 (2004) 